\title{
Asymptotic stability of linearly evolving non-stationary modes in a uniform hydromagnetic field over a rotating-disk flow with suction and injection
}

\author{
M TURKYILMAZOGLU \\ Mathematics Department, University of Hacettepe, 06532-Beytepe, Ankara, \\ Turkey \\ e-mail: turkyilm@hotmail.com
}

MS received 1 April 2009; revised 29 June 2009

\begin{abstract}
In this paper the linear stability properties of the magnetohydrodynamic flow of an incompressible, viscous and electrically conducting fluid are investigated for the boundary-layer due to an infinite permeable rotating-disk. The fluid is subjected to an external magnetic field perpendicular to the disk. The interest lies also in finding out the effects of uniform suction or injection. In place of the traditional linear stability method, a theoretical approach is adopted here based on the high-Reynolds-number triple-deck theory. It is demonstrated that the nonstationary perturbations evolve in accordance with an eigenrelation analytically obtained.
\end{abstract}

Keywords. Rotating disk; stability analysis; hydromagnetic effects; suction/injection

\section{Introduction}

Means of stabilization of fully three-dimensional boundary-layer flows particularly those occurring over aerodynamic body shapes have been the intense focus of past and recent studies. One of the unique prototype model flows whose stability nature exhibits close similarities with the stability properties of other fully three-dimensional boundary-layer flows of reallife applications is the well-known rotating-disk boundary-layer flow due to Kármán (1921). The complete understanding of the stability of the Karman self-similar exact solution of the Navier-Stokes equations for the flow over a rotating-disk is therefore received a special attention and it is believed to shed important light on the unresolved problems of physical significance such as the laminar-turbulent transition phenomenon of the swept-back wing aircraft. The present work is hence devoted to the rotating-disk flow instabilities under the influence of a uniform normal magnetic field and also uniform suction applied through the surface of the disk.

When the fluid is non-conducting, the experimental, theoretical and numerical results point commonly to the destabilization mechanism by means of crossflow vortex instability caused mainly because of inflectional nature of Karman mean-flow. Research particularly making 
use of the parallel-flow assumption also demonstrated that this instability may be cast into convective type as well as absolute type, both of which result in the form of viscous lowerbranch or inviscid upper-branch. The early works in this area, such as Gregory et al (1955); Malik et al (1981); Malik \& Poll (1985); Malik (1986) and Balakumar \& Malik (1990) highlighted the convective instability features, while the research conducted by Lingwood (1995), Cole (1995), Lingwood (1996) and Turkyilmazoglu (1998) Turkyilmazoglu et al (2000); Turkyilmazoglu \& Gajjar (2001a), Turkyilmazoglu \& Uygun (2005) investigated the absolute instability of the crossflow vortices. The non-linear effects and secondary instability of the primary absolute instability was also studied in Pier (2003). Although this newly discovered mechanism is suggested by the above researchers to give rise to a route to transition to turbulence, this proposition is not fully supported by the numerical simulation study of Davies \& Carpenter (2003) (and also the recent experiments of Othman (2005) and Othman \& Corke (2005)), which showed that the local absolute instability does not lead to a global instability mode.

On the other hand, when the fluid is conducting within the presence of a magnetic field applied in any direction to the main stream of the boundary-layer flow, the stability investigations of the rotating-disk boundary-layer flows are limited to a few in number. Instead, mainly due to the practical interest in machinery such as gas-turbine rotors, in industrial applications such as electromagnetic stirring of liquid metals, as well as in a variety of astrophysical and geophysical situations, the steady or unsteady viscous conducting Karman basic flow was extensively computed by numerical and asymptotical means by researchers. For instance, Sparrow \& Gregg (1959) and Sparrow \& Cess (1962) examined the effects of magnetic field applied normal to the disk and also heat transfer on the steady Karman mean-flow. The unsteady extension of this work was later accomplished by Benton (1966) and recently by Attia (2003). A universal outcome of these studies is that the presence of a transverse magnetic field leads to a significant decrease in the magnitudes of the flow quantities in the boundary-layer of a rotating-disk flow. Unlike the case of a normal magnetic field, the presence of a circular magnetic field in the flow of an incompressible viscous conducting fluid over a rotating-disk yields a reduction in the axial flow variable together with a thickening influence on the boundary-layer, as demonstrated by Pao (1968).

Hydrodynamic and hydromagnetic stability of boundary-layer flows, see, for instance, Chandrasekkar (1961), Bhatnagar \& Banerjee (1978), Chang (2003), David et al 2005) and Kafoussias \& Nanousis (1999) are significant from the mathematical as well as the practical applications point of view. It seems that in the literature there is a lack of studies on the stability analysis of the viscous rotating-disk boundary-layer flow when a magnetic field is present in the fluid. This might be owing to the reason that the reduction of the mean-flow velocities in the case of a transverse magnetic field, for instance, enables one to think of the stabilization effect of the conducting fluid. However, the full stability characteristics and the absolute influence of magnetic field on the convective/absolute instability mechanism have not yet been fully demonstrated to the best of the knowledge of the author. The only exception is the recent study of Jasmine \& Gajjar (2005) (which appeared during the preparation stage of the present work) in which it is concluded that the existence of the normal magnetic field acts indeed as to stabilize both the convective as well as absolute type of instability mechanisms; further, the higher the strength of the magnetic field, the stronger the stabilization, as physically expected. It is believed, although to be verified in real-life situations, the results of the present work will enable to gain new understanding to the problem of laminar-turbulent transition.

In the present paper, we consider the rotating-disk boundary-layer flow in an electrically conducting fluid in the presence of a magnetic field applied in the direction normal to the disk 
surface, similar to the work of Jasmine \& Gajjar (2005), but with the inclusion of suction or injection through the rigid wall of the disk. In addition to this, our present research also differs from that of Jasmine \& Gajjar (2005) in place of the traditional linear stability analysis under the assumption of a usual parallel-flow approximation, a more rigorous mathematical approach is pursued in this study accounting for the non-parallelism of the fluid motion as opposed to the above-mentioned technique. This approach is based on the viscous-inviscid interactions of the boundary-layer with the external flow field regarding the large-Reynoldsnumber assumption and is termed as the triple-deck theory. Having been formally introduced first by Stewartson (1969); Stewartson \& Williams (1969), the theory was successfully applied to analyse asymptotically the linear and weakly non-linear stability features of the stationary crossflow vortex modes of the Karman boundary-layer flow in the studies of Hall (1986), Mackerrel (1987), Bassom \& Gajjar (1988) and Seddougui (1990). From these works, the non-parallelism and non-linearity are in general found to be destabilizing. The effects arising from the non-zero-frequency waves were later analysed by Turkyilmazoglu (2007) and Turkyilmazoglu \& Gajjar (1999). It was comprehensively concluded in the last studies that the non-stationary modes are more destabilized than the stationary modes and also the compressibility influences contribute to the stabilization of the flow. The impacts of applying suction or injection through the disk surface were discussed for the absolute instability in the work of Lingwood (1997) and for the convective instability in the works of Turkyilmazoglu (2005), Bassom \& Seddougui (1992) and Seddougui \& Bassom (1996). The latter study also demonstrated clearly the stabilization effect of suction and the reverse effect of injection.

Thus, in the present work the focuss is on the convective instability of the lower-branch modes and aim to determine the analytical structure of these modes as in the work of Turkyilmazoglu (2007) but this time with the consideration of a conducting fluid. To derive a self-similar form for the mean-flow we make use of the assumptions that the effects of the electric field are of no significance and that the fluid motion cannot alter the applied magnetic field, being constant everywhere. The only effect of the magnetic field on the boundary-layer flow is the presence of Lorentz force which appears in the momentum equations with an extra forcing term. It is further presumed that the magnetic field is under the strong influence of diffusive effects within the presence of a low magnetic-field Reynolds number. Taking these into consideration a derivation for the eigenrelation is achieved governing the stability features of the non-stationary modes. Analysing this relation enables us to closely deduce the effects of not only the magnetic field but also the suction and injection influences on the disturbances. Indeed, a numerical treatment of this equation clearly demonstrates the stabilizing impact of the presence of a transverse magnetic field no matter the level of suction or injection, even though the injection tends to destabilize the boundary-layer. Whatever the case, the non-stationary positive-frequency waves are found to be more dangerous as compared to the stationary or non-stationary disturbances having negative frequencies, in parallel to the outcomes of Turkyilmazoglu (2007) and Turkyilmazoglu (2005) in the case of a non-magnetic medium. Meanwhile, the wavenumber and wave-angle in the limit of large-Reynolds-numbers computed from the asymptotic eigenrelation at several magnetic-field parameters compare fairly well with the numerical solution of the full stability equations arising from the parallelflow assumption. Comparison with the parallel modes also signifies destabilization due to the non-parallelism accounted for in the asymptotic analysis. In addition to this, the appearance of double modes for the lower-branch curves having positive frequencies as first encountered in the numerical stability solution of non-stationary incompressible flow in Balakumar \& Malik (1990) is shown here, whose interval of occurrence is particularly increased in the case of suction, in a more pronounced manner with increasing magnetic field. 
The paper is organized as follows. Section 2 presents the partial differential equations governing the stability of the viscous conducting boundary-layer fluid flow over a rotatingdisk together with the basic steady Karman flow including the suction/blowing on the disk surface. Section 3 involves the construction of a set of expansions and their solutions describing the flow character especially in the wall-layer region and also matching with the other two asymptotic regions within the concept of triple-deck theory. Section 4 contains several results. First, the mean-flow results are presented in $4 \cdot 1$. Second, in 4.2 the evolution of the incompressible lower-branch modes and the effects of magnetic field as well as suction/blowing are presented from an obtained eigenrelation that connects the effective wavenumber, waveangle and frequency of the stability modes, and the findings calculated here are compared with the ones computed using the conventional linear stability theory together with the parallelflow approximation in $\$ 4 \cdot 3$. Finally, our conclusions are preceded in $\$ 5$.

\section{Problem formulation}

\subsection{Governing equations of the flow}

We are concerned here with the three-dimensional, unsteady flow of an incompressible, electrically conducting viscous fluid over an infinite disk rotating with a constant angular velocity $\Omega_{a}$ about its axis of rotation, the axial axis $z$. We also assume that in the direction normal to the disk surface a uniform magnetic field $B=B_{0}$ is applied. The flow variables are non-dimensionalized based on a length scale $l$, such as the local radius of the disk and also a velocity scale $U_{a}=l \Omega_{a}$, being the free-stream azimuthal velocity. Hence, throughout the analysis, the cylindrical coordinates $(r, \theta, z)$ are made dimensionless with respect to the length scale $l$ (except the azimuthal variable $\theta$ ), the time variable $t$ with $\frac{l}{U_{a}}$, the velocities $\mathbf{u}=(u, v, w)$ with $U_{a}$, the pressure $p$ with $\rho U_{a}^{2}, \rho$ being the constant density and the magnetic field $\mathbf{B}$ with $B_{0}$. Thus, relative to this non-dimensional cylindrical polar coordinates $(r, \theta, z)$ which possess an orthonormal unit base $(\hat{r}, \hat{\theta}, \hat{k})$ and also rotate with the disk, the unsteady magnetohydrodynamic equations governing the viscous fluid flow are given as

$$
\begin{aligned}
& \nabla \cdot \mathbf{u}=0 \\
& \frac{\partial \mathbf{u}}{\partial t}+(\mathbf{u} \cdot \nabla) \mathbf{u}+2(\hat{k} \times \mathbf{u})-\mathrm{r} \hat{r}=-\nabla p+m \mathbf{J} \times \mathbf{B}+\frac{1}{R} \nabla^{2} \mathbf{u} \\
& \frac{\partial \mathbf{B}}{\partial t}=\nabla \times(\mathbf{u} \times \mathbf{B})+\frac{1}{R_{m}} \nabla^{2} \mathbf{B} \\
& \nabla \cdot \mathbf{J}=0 \\
& \nabla \cdot \mathbf{B}=0 \\
& \nabla \times \mathbf{E}=-\frac{\partial \mathbf{B}}{\partial t} .
\end{aligned}
$$

Here, $\nabla^{2}$ is the usual Laplacian operator in cylindrical coordinates, $R$ defines the characteristic Reynolds number given by $R=\frac{\Omega_{a}^{2} l}{v}$ with $v$ the kinematic viscosity of the fluid, $R_{m}$ defines the magnetic Reynolds number given by $R=\sigma \mu l^{2} \Omega_{a}$ with $\sigma$ the electrical conductivity of the fluid and $\mu$ the free-space magnetic permeability, and also $m$ is the magnetic 
interaction parameter defined by $m=\frac{\sigma B_{0}^{2}}{\rho \Omega_{a}}$ and assigned a positive number. In addition to this, the current density $\mathbf{J}$ is given by $\mathbf{J}=\mathbf{E}+\mathbf{u} \times \mathbf{B}$ and also the magnetic Reynolds number is assumed to be much smaller than the hydrodynamic Reynolds number, which is the case for several practical situations. Thus, it can be assumed that due to the low magnetic Reynolds number the applied magnetic field is unaffected by the effect of the motion of the conducting fluid. Whereas, the effect of the magnetic field on the fluid motion manifests itself in the form $m \mathbf{J} \times \mathbf{B}$ on the right-hand side of the momentum equations (2), which is known as the Lorentz force, the components of which can be evaluated as $(-u,-v, 0)$ for a normal electric field. It is thus further assumed that there are no radial or azimuthal currents influencing the motion of the fluid flow.

\subsection{Basic steady flow}

To obtain the steady incompressible conducting self-similar flow equations of motion, which are known as the Karman similarity solution in the non-conducting case, equations (1-6) must be supplemented with the no-slip boundary conditions on the wall except with suction/injection permitted on the wall-normal velocity through a scaled parameter $\bar{s}$ such that $\bar{s}>0$ denotes the suction and $\bar{s}<0$ the blowing applied to the flow at the surface of the disk. Additionally, no motion far away from the disk should be allowed. From now on the Reynolds number is assumed to be large and since the boundary-layer thickness is of order of magnitude $R^{-1 / 2}$, the steady incompressible boundary-layer flow in the presence of a uniform transverse magnetic field over a rotating-disk evolves along a boundary-layer coordinate of order unity, defined by $y=R^{1 / 2} z$, as for the non-magnetic case. Then the mean-flow quantities take the form

$$
\mathbf{u}_{\mathbf{B}}=\left(r \bar{u}(y), r \bar{v}(y), R^{-1 / 2} \bar{w}(y), \frac{1}{R} \bar{p}(y)\right) .
$$

Substituting the above into the Navier-Stokes equations (1-6), the functions $\bar{u}, \bar{v}$ and $\bar{w}$ representing the basic flow can be shown to satisfy

$$
\begin{aligned}
& \psi^{\prime 2}-(\bar{v}+1)^{2}-2 \psi \psi^{\prime \prime}-\psi^{\prime \prime \prime}+m \psi^{\prime}=0, \\
& 2 \psi^{\prime}(\bar{v}+1)-2 \psi \bar{v}^{\prime}-\bar{v}^{\prime \prime}+m(\bar{v}+1)=0,
\end{aligned}
$$

for an appropriately defined stream function variable $\psi$ given as $\bar{u}=\psi^{\prime}$ and $\bar{w}=-2 \psi$. Equations (8) are to be solved subject to

$$
\begin{array}{lll}
\psi=\frac{\bar{s}}{2}, & \psi^{\prime}=0, \quad \bar{v}=0, & \text { at } y=0, \\
\psi^{\prime}=0, & \bar{v}=-1, \quad \text { as } y \rightarrow \infty .
\end{array}
$$

It is clear that equations (8-9) reduce to the usual Karman equations in the non-magnetic case, as extensively used in the literature, see, for example, Hall (1986) and Malik (1986). The mean-flow equations of the conducting fluid (8-9) were also used in the context of finding similarity solutions under the influence of magnetic-resonance parameter $m$ for a rotating-disk boundary-layer flow, see, for example, Sparrow \& Cess (1962) and Hossain et al (2001). The ensuing large-Reynolds-number asymptotic analysis requires the values of $\bar{u}^{\prime}(0)$ and $\bar{v}^{\prime}(0)$ for varying magnetic field as well as suction parameters, which are numerically calculated from equations (8-9) and discussed in detail in section 4.1. 


\section{Disturbance equations and asymptotic analysis}

Since we are concerned with the hydrodynamic linear stability of the magnetohydrodynamic motion described by equations (1-6), the instantaneous dimensionless flow field is decomposed into a mean-flow determined from equations (7-9) plus infinitesimally small perturbations represented as

$$
\tilde{U}(r, \theta, z, t), \tilde{V}(r, \theta, z, t), \tilde{W}(r, \theta, z, t), \tilde{P}(r, \theta, z, t) .
$$

Substituting next the mean-flow together with these disturbances into the governing NavierStokes equations (1-6), subtracting out the basic field, and linearizing with respect to the perturbations, the evolution of the incompressible disturbed flow under the influence of the magnetic-interaction parameter $m$ will be governed by a system of equations, which are implicitly dependent on the suction parameter $\bar{s}$, and are given by

$$
\begin{aligned}
& \frac{\partial \tilde{U}}{\partial r}+\frac{1}{r} \frac{\partial \tilde{V}}{\partial \theta}+\frac{\tilde{U}}{r}+\frac{\partial \tilde{W}}{\partial z}=0, \\
& \left(L+\bar{u}+m-R^{-1} L_{1}\right) \tilde{U}-2(\bar{v}+1) \tilde{V}+r \frac{d \bar{u}}{d z} \tilde{W}=-\frac{\partial \tilde{P}}{\partial r}, \\
& \left(L+\bar{u}+m-R^{-1} L_{1}\right) \tilde{V}+2(\bar{v}+1) \tilde{U}+r \frac{d \bar{u}}{d z} \tilde{W}=-\frac{1}{r} \frac{\partial \tilde{P}}{\partial \theta}, \\
& \left(L+R^{-1 / 2} \frac{d \bar{w}}{d z}-R^{-1} L_{1}\right) \tilde{W}=-\frac{\partial \tilde{P}}{\partial z},
\end{aligned}
$$

where, the partial differential operators $L$ and $L_{1}$ are respectively, defined by $L=\frac{\partial}{\partial t}+$ $r \bar{u} \frac{\partial}{\partial r}+\bar{v} \frac{\partial}{\partial \theta}+R^{-1 / 2} \bar{w} \frac{\partial}{\partial z}$ and $L_{1}=\frac{\partial^{2}}{\partial z^{2}}+\frac{\partial}{\partial z}$. It should be noticed that only the terms which are of significance for the subsequent asymptotic analysis are retained in the above equations. In addition to equations (10) conditions of no-slip at the wall and disappearing at the infinity on the disturbances are imposed.

An order-of-magnitude analysis balancing the viscous, convective and pressure forces in equations (1-6) results in a triple-deck region as in Hall (1986) and displayed in figure 1, whose thicknesses are found to be $O\left(\varepsilon^{9}\right)$ for the viscous lower-deck region III, $O\left(\varepsilon^{8}\right)$ for the main-deck region II and $O\left(\varepsilon^{4}\right)$ for the inviscid upper-deck region I. The small parameter $\varepsilon$ is defined as $\varepsilon=\frac{1}{R^{1 / 16}}$.

The intention hereafter is to determine the behaviour of the incompressible disturbances within the influence of a normal magnetic field but having long time scale, small frequencies and evolving near the lower-branch curve of the stability, and also to explore the possible impacts of the suction or injection. As in Hall (1986) an effective velocity profile can be defined by $\alpha r \bar{u}+\beta \bar{v}$, with $\alpha$ and $\beta$ denoting wavenumbers respectively, in the $r$ and $\theta$ directions. Moreover, defining the frequency of the disturbances as $\omega$, the wavenumbers and frequency are expanded in terms of the small parameter $\varepsilon$ in following manner

$$
\begin{gathered}
\alpha=\alpha_{0}+\varepsilon^{2} \alpha_{1}+\cdots, \\
\beta=\beta_{0}+\varepsilon^{2} \beta_{1}+\cdots, \\
\omega=\varepsilon \omega_{0}+\varepsilon^{2} \omega_{1}+\cdots .
\end{gathered}
$$




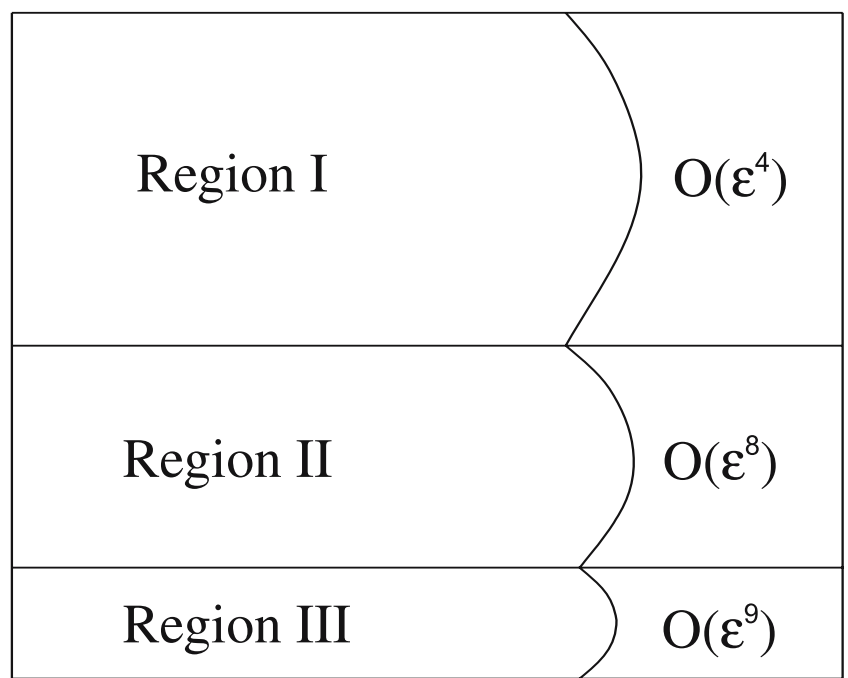

\section{$\mathrm{O}\left(\varepsilon^{4}\right)$}

Figure 1. Asymptotic regions of the triple-deck and thicknesses of each layer are demonstrated in terms of a small parameter $\varepsilon=R^{-1 / 16}$ for the rotatingdisk boundary-layer flow. Here, region I corresponds to the upperdeck, region II to main-deck and region III to lower-deck.

Our ultimate purpose is to find the local wavenumber and frequency components in (11) which lead to a neutrally stable flow at a radial location $r$ for the non-stationary modes, as implemented in Hall (1986) for the stationary ones. Thus, using the normal-mode analysis, the structure of the perturbations is taken in the form

$$
(\tilde{U}, \tilde{V}, \tilde{W}, \tilde{P})=(U(z), V(z), W(z), P(z)) e^{\left(i / \varepsilon^{4}\right)\left(\int \alpha(r) d r+\beta \theta-\varepsilon^{2} \omega t\right)} .
$$

Making a substitution of the wavenumbers and frequency as given in (11) together with the disturbances in (12) into the linearized incompressible Navier-Stokes equations in (10), the expansions as well as the solutions turn out to be unaffected and are the same as those previously found in Turkyilmazoglu (2007) for the zero-suction and non-magnetic case in the upper and main decks as depicted in figure 1. The contributions arising from the nonzero magnetic-interaction parameter $m$ start influencing the disturbance field at the next level, which is redundant for the ensuing analysis. It is further convenient to define here the effective zeroth-order wavenumber $\gamma_{0}$ as

$$
\gamma_{0}^{2}=\alpha_{0}^{2}+\frac{\beta_{0}^{2}}{r^{2}}
$$

Moreover, allowing the shear stress at the wall to disappear in the main-deck enables us to express an eigenrelation for the leading-order eigenvalues through the equation

$$
\frac{\alpha_{0} r}{\beta_{0}}=-\frac{\bar{v}^{\prime}(0)}{\bar{u}^{\prime}(0)} .
$$

This relation is yet to be computed for varying magnetic-field strength parameter $m$ as well as the suction parameter $\bar{s}$, whose corresponding constant value of 1.207 was given in Hall (1986) for zero-suction and non-conducting incompressible flow motion case. Dissimilar to the case 
of stationary modes as considered in Hall (1986) is, too, the occurrence of a wall-normal perturbation shift in the boundary-layer here for the non-stationary perturbations through the leading-order frequency term $\omega_{0}$, that is successfully tackled below by means of the lowerdeck region.

We next pass to region III which is needed to satisfy the zero-slip condition on the disk surface and is comparably more significant than the other regions, due to the fact of the resultant eigenrelation for the neutral modes within this region. As previously stated, the thickness of this viscous sublayer region is given as

$$
\zeta=\varepsilon^{-9} z=O(1)
$$

and the Taylor expansion of the basic flow quantities (7) in terms of this scaling gives rise to

$$
\begin{aligned}
& \bar{u}=\varepsilon \bar{u}_{0} \zeta+\varepsilon^{2} \bar{u}_{1} \zeta^{2}+\cdots, \\
& \bar{v}=\varepsilon \bar{v}_{0} \zeta+\varepsilon^{2} \bar{v}_{1} \zeta^{2}+\cdots \\
& \bar{w}=-\bar{s}-\varepsilon^{2} \bar{u}_{0} \zeta^{2}-\frac{2}{3} \varepsilon^{3} \bar{u}_{1} \zeta^{3}+\cdots
\end{aligned}
$$

Expanding the solutions found in region II in terms of the thickness of region III evidently implies that the expansions for the lower-deck perturbations should be sought in the form

$$
\begin{aligned}
& U=\varepsilon^{-1} r A\left[\bar{u}_{0}+2 \varepsilon \bar{u}_{1} \zeta+\cdots\right]+\varepsilon^{-1} U_{-1}+U_{0}+\varepsilon U_{1}+\cdots \\
& V=\varepsilon^{-1} r A\left[\bar{v}_{0}+2 \varepsilon \bar{v}_{1} \zeta+\cdots\right]+\varepsilon^{-1} V_{-1}+V_{0}+\varepsilon V_{1}+\cdots \\
& W=-i A\left[\varepsilon^{5}\left(\alpha_{0} r \bar{u}_{1}+\beta_{0} \bar{v}_{1}\right) \zeta^{2}+\cdots\right]+\varepsilon^{6} W_{0}+\cdots \\
& P=\varepsilon^{3} P_{0}+\cdots
\end{aligned}
$$

with $A$ being defined as $A=\frac{\tilde{\gamma}_{0} C}{\beta_{0}^{2}}$.

Having substituted expressions (13-14) into the linearized disturbance equations (10), the successive-order equations from the continuity, radial and normal momentum equations read as follows

$$
\begin{aligned}
& i\left(\alpha_{0} r U_{-1}+\beta_{0} V_{-1}\right)=0, \\
& i\left(\alpha_{0} r U_{0}+\beta_{0} V_{0}\right)=0, \\
& i\left(\alpha_{0} r U_{1}+\beta_{0} V_{1}\right)+r \frac{\partial W_{0}}{\partial \zeta}=-i A r U_{10}-i\left(\alpha_{1} r U_{-1}+\beta_{1} V_{-1}\right), \\
& \frac{\partial^{2} U_{-1}}{\partial \zeta^{2}}-i U_{01} \zeta^{2} U_{-1}=0, \\
& \frac{\partial^{2} U_{0}}{\partial \zeta^{2}}-i U_{01} \zeta^{2} U_{0}=r \bar{u}_{0} W_{0}+i\left(U_{10} \zeta-\omega_{0}\right)\left(A r \bar{u}_{0}+U_{-1}\right)+i U_{02} \zeta^{3} U_{-1}-\bar{s} \frac{\partial U_{-1}}{\partial \zeta}
\end{aligned}
$$




$$
\begin{aligned}
& \frac{\partial^{2} U_{1}}{\partial \zeta^{2}}-i U_{01} \zeta^{2} U_{1}=2 r \bar{u}_{1} \zeta W_{0}+r \bar{u}_{0} W_{1}+i \alpha_{0} P_{0}-2 V_{-1}+i U_{11} \zeta^{2}\left(A r \bar{u}_{0}+U_{-1}\right) \\
& \quad+i\left(U_{10} \zeta-\omega_{0}\right)\left(2 A r \bar{u}_{1} \zeta+U_{0}\right)+i U_{02} \zeta^{3} U_{0}+i U_{20} \zeta\left(r A \bar{u}_{0}+U_{-1}\right)+i U_{03} \zeta^{4} U_{-1} \\
& \quad-\bar{s} \frac{\partial U_{0}}{\partial \zeta}+m\left(r A \bar{u}_{0}+U_{-1}\right) \\
& \frac{\partial P_{0}}{\partial \zeta}=0
\end{aligned}
$$

The solution to the leading-order pressure $P_{0}$ is obtained as $P_{0}=C(r)$. Because the equations corresponding to azimuthal velocity expansion are similar to (16), they are omitted here. In the above equations $U_{i j}$ is defined by $U_{i j}=\alpha_{i} r \bar{u}_{j}+\beta_{i} \bar{v}_{j} ; i, j=0-3$. It is worth noting here that the term $U_{-1}$ (and also $V_{-1}$ in the azimuthal momentum equation) appearing on the righthand sides of equations (15-16) is due to the Coriolis effects, showing that the structure of the neutral modes of small-frequency waves depends upon both viscous and Coriolis effects as for the non-magnetic case. In addition to this, equations turn out to be same as those found in Hall (1986) when the suction parameter and the magnetic-field parameters are omitted.

It is found after a regular matching is carried out with the main-deck solutions that the boundary conditions for the perturbation terms $U_{-1}$ and $V_{-1}$ given in the expansion (14) are $U_{-1}=-r \bar{u}_{0} A, V_{-1}=-r \bar{v}_{0} A \quad$ at $\quad \zeta=0, \quad U_{-1}=V_{-1}=0 \quad$ as $\quad \zeta \rightarrow \infty$,

and hence the leading-order disturbance terms have the solutions of the form

$$
\begin{aligned}
& U_{-1}=-\operatorname{Ar} \bar{u}_{0} \frac{U_{c}(0, \sqrt{2} s)}{U_{c}(0,0)}, \\
& V_{-1}=-\operatorname{Ar} \bar{v}_{0} \frac{U_{c}(0, \sqrt{2} s)}{U_{c}(0,0)},
\end{aligned}
$$

with $s=\delta^{1 / 4} \zeta, \delta=i U_{01}$. Here $U_{c}$ denotes parabolic cylinder function, asymptotically decaying at far distances from the wall, see, for instance, Abramowitz \& Stegun (1955). After a similar manipulation as implemented in Hall (1986) and Turkyilmazoglu (2007), $W_{0}$ is shown to satisfy the following ordinary differential equation

$$
\begin{aligned}
W_{0}^{\prime \prime \prime}-s^{2} W_{0}^{\prime}+2 s W_{0}= & \delta^{-3 / 4}\left\{\gamma_{0}^{2} C-2 i \frac{\beta_{0}}{r}\left[1+\frac{\bar{v}_{0}^{2}}{\bar{u}_{0}^{2}}\right] U_{-1}\right. \\
& \left.-i A \delta^{3 / 4} s\left(U_{10} \delta^{-1 / 4} s-2 \omega_{0}\right)\right\},
\end{aligned}
$$

together with the boundary conditions as a result of matching with the main-deck

$$
W_{0}(0)=0, \quad W_{0}(\infty)=-A\left(U_{10} \zeta-i \omega_{0}\right),
$$

whose solution using equation (19) can be written in the form

$$
\begin{aligned}
W_{0}= & -i A U_{10} \delta^{-1 / 4} s+i A \omega_{0} F_{1}(s) \\
& +\delta^{-3 / 4}\left\{\gamma_{0}^{2} C F_{2}(s)+2 i \beta_{0} A\left[1+\frac{\bar{v}_{0}^{2}}{\bar{u}_{0}^{2}}\right] \frac{\bar{u}_{0}}{U_{c}(0,0)} F_{3}(s)\right\}+O\left(\zeta^{2}\right),
\end{aligned}
$$


where, the last term can be determined after a matching with the main-deck. The particular solutions $F_{i}, i=1-3$ are forced to satisfy the boundary conditions (21) and the corresponding differential equations are given in Turkyilmazoglu (2007). It is clear from (16) that the solution to $U_{1}$ and $V_{1}$ depends explicitly on the magnetic-field parameter $m$ but with the help of vanishing shear stress at the wall the removal of the explicit dependence of $W_{0}$ on $m$ becomes possible. Moreover, for the subsequent analysis, the values of the following parameters can be obtained after a straightforward computation as

$$
\begin{aligned}
& I_{1}=F_{1}^{\prime}(0)=1 \cdot 3520, \\
& I_{2}=F_{2}^{\prime}(0)=\frac{1}{2 U_{c}(0,0)} \int_{0}^{\infty} \theta U_{c}(0, \theta) d \theta=0.5990, \\
& I_{3}=2 \frac{F_{3}^{\prime}(0)}{U_{c}(0,0)}=\frac{1}{U_{c}^{2}(0,0)} \int_{0}^{\infty} \theta U_{c}^{2}(0, \theta) d \theta=0.4570 .
\end{aligned}
$$

\section{Results and discussion}

\subsection{The mean-flow}

A numerical integration of equations (7-9) was implemented first to obtain the basic flow quantities for a range of values $m$ and $\bar{s}$. Figures 2-3 (a-d) shows the basic velocity profiles for a variety of suction/injection parameters at magnetic and some non-magnetic flow cases. It is seen that the presence of normal magnetic field acts to reduce the mean-flow velocities, while injection increases the boundary-layer thickness and pushes the inflexion points away from the wall. The decrease in boundary-layer thickness is much felt in the case of suction applied. The perturbation approach of small magnetic-field parameter as outlined in the appendix can also be seen in figure 2(d) by the starred curves. Figure 3(d) shows the mean-flow profiles evaluated at a large magnetic-field parameter $m=5$. The smooth curves correspond to numerical parts, and the starred ones to asymptotic ones from (24) below. The behaviour of the mean-flow quantities as depicted in figures 2-3 will ultimately determine the stability of the flow.

Asymptotic solutions for the large-suction or blowing cases may be obtained as in Stuart (1954) with fixed and finite magnetic-field parameter, but we prefer here large magnetic field effect on the solution for fixed and finite-suction parameter. It can be seen that for strong magnetic field, that is $m \gg 1$, the basic flow profile finds itself confined to a layer adjacent to the wall of thickness $O\left(m^{-1 / 2}\right)$. Thus, writing $v=m^{1 / 2} y$, where $v$ is $O(1)$, the mean-flow quantities can be expanded as

$$
\begin{aligned}
& F=m^{-1} F_{0}+m^{-3 / 2} F_{1} \cdots, \quad G=G_{0}+m^{-1 / 2} G_{1}+\cdots, \\
& H=-\bar{s}+m^{-3 / 2} H_{0}+m^{-2} H_{1}+\cdots .
\end{aligned}
$$

Thus, solving for the leading and second-order terms it is found that

$$
\begin{aligned}
& F_{0}=\frac{1}{3}\left(e^{-v}-e^{-2 v}\right), \quad G_{0}=-1+e^{-v}, \quad H_{0}=-\frac{1}{3}+\frac{2}{3}\left(e^{-v}-\frac{e^{-2 v}}{2}\right), \\
& F_{1}=-\frac{7 \bar{s}}{18}\left(e^{-v}-e^{-2 v}\right)-\frac{\bar{s}}{6} v\left(e^{-v}-2 e^{-2 v}\right), \quad G_{1}=-\frac{\bar{s}}{2} v e^{-v},
\end{aligned}
$$




$$
H_{1}=\frac{5 \bar{s}}{9}-\frac{7 \bar{s}}{9}\left(e^{-v}-\frac{e^{-2 v}}{2}\right)-\frac{\bar{s}}{3}\left[(1+v) e^{-v}-\frac{(1+2 v) e^{-2 v}}{2}\right] .
$$

The derivatives of the wall values of mean-flow quantities as a result of (24) are thus calculated in terms of the fixed suction parameter $\bar{s}$ as

$$
\bar{u}_{0}=\frac{m^{-1 / 2}}{3}\left(1-\frac{2 \bar{s}}{9} m^{-1 / 2}\right), \quad \bar{v}_{0}=-m^{1 / 2}\left(1+\frac{\bar{s}}{2} m^{-1 / 2}\right) .
$$

For comparison purposes with the exact numerical value of vertical velocity at infinity $h_{\infty}$, the corresponding asymptotic value from the above relations can be extracted as

$$
h_{\infty a}=-\bar{s}-m^{-3 / 2} / 3+m^{-2} \frac{5 \bar{s}}{9} .
$$

The effects of the magnetic-field parameter $m$ are presented in tables $1-3$ on the parameters $\bar{u}_{0}, \bar{v}_{0}, h_{\infty}$ and the ratio $r a=-\frac{\bar{v}_{0}}{\bar{u}_{0}}$ for changing suction or injection cases. Table 1 is for the non-magnetic case. As tabulated in tables, the values $\bar{u}_{0}, \bar{v}_{0}, h_{\infty}$ and $r a$ strongly affected by the presence of a normal magnetic field. It can be observed that the mean-flow values decrease much as the magnetic-field parameter gets larger, whereas an opposite effect is seen for $r a$.

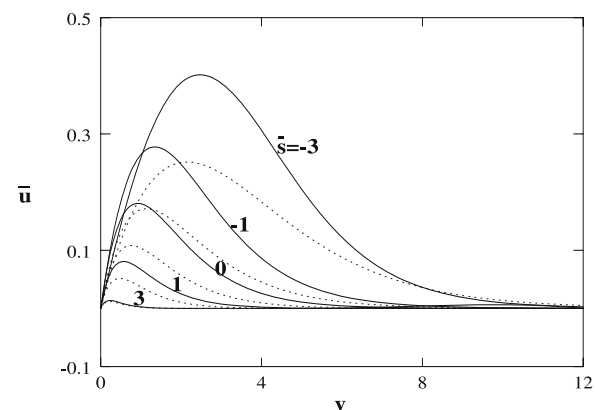

(a)

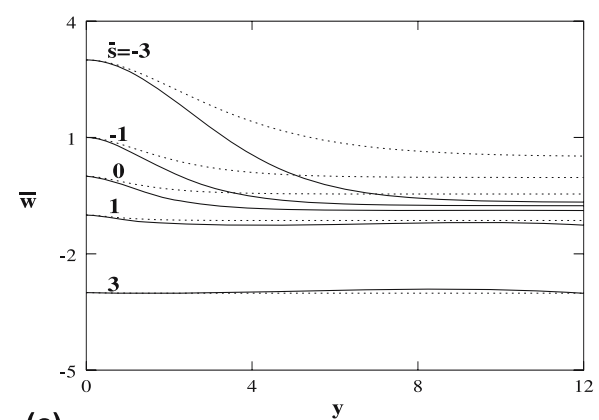

(c)

Figure 2. Basic flow quantities for the rotating-disk flow are shown with magnetic-strength parameters $m=0$ (continuous curves) and $m=0.5$ (dotted curves) for several values of suction parameter $s$, respectively in (a) the radial velocity profiles, (b) the circumferential velocity profiles, (c) the wall-normal velocity profiles, (d) shows the effect of small magnetic field $m=0.5$ as calculated from (7) (smooth curves) and also from the perturbation solution as outlined in the appendix (starred curves) for a suction and a blowing case.

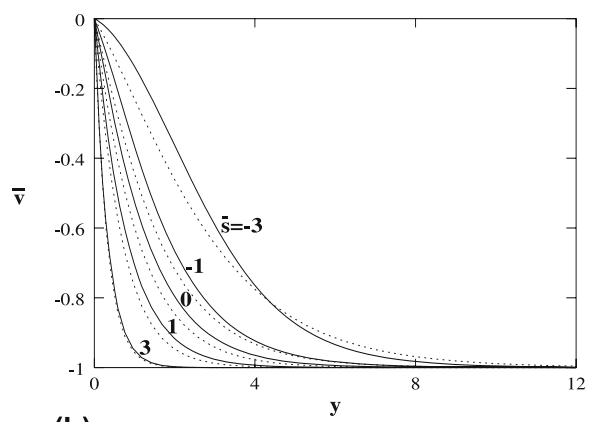

(b)

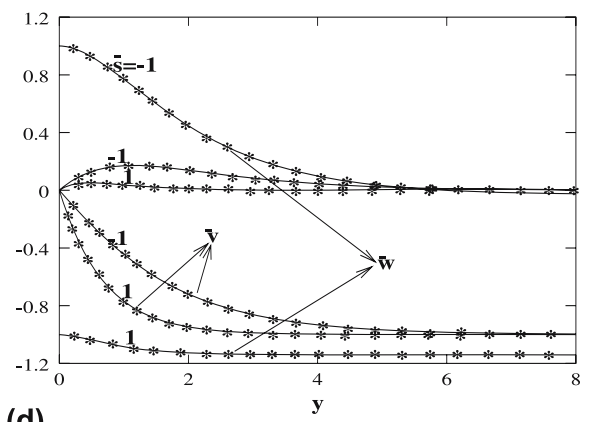

(d) 


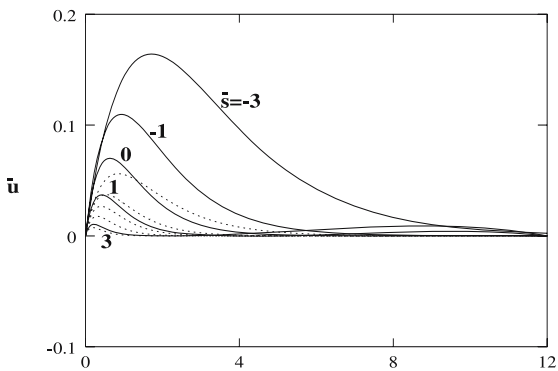

(a)

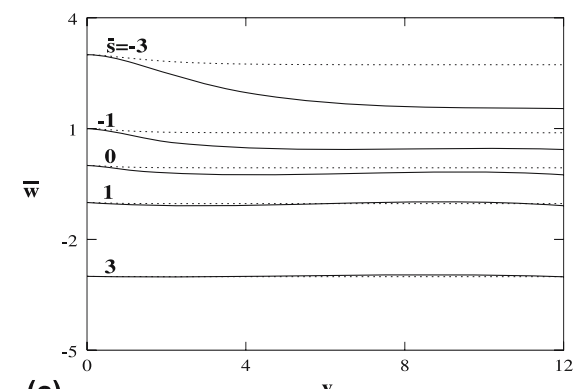

(c) $\mathbf{y}$

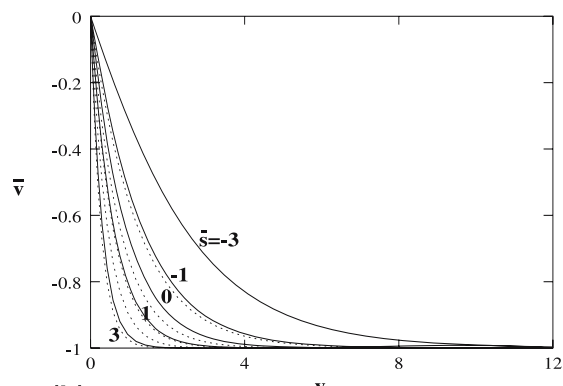

(b)

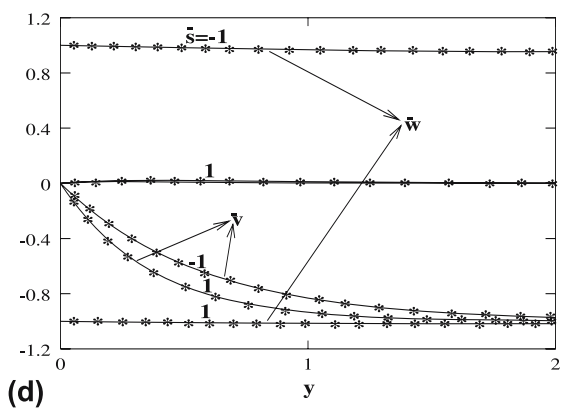

Figure 3. Basic flow quantities for the rotating-disk flow are shown with magnetic-strength parameters $m=1$ (continuous curves) and $m=3$ (dotted curves) for several values of suction parameter $s$, respectively in (a) the radial velocity profiles, (b) the circumferential velocity profiles, (c) the wall-normal velocity profiles, (d) shows the effect of large magnetic field $m=5$ as calculated from (7) (smooth curves) and also from (24) (starred curves) for a suction and a blowing case.

Tables also show the asymptotic correspondence of $r a$, denoted by ras as well as the asymptotic correspondence of $h_{\infty}$, denoted by $h_{\infty a}$ and computed from the asymptotic values (25-26). The parameter $r a$ is closely related to the modes having zero shear at the wall and they also give the leading-order orientation of the vortices $\epsilon=\arctan \left(\frac{\beta_{0}}{\alpha_{0} r}\right)$. A close agreement between the exact and asymptotic values of $h_{\infty}, r a$ and $h_{\infty a}, r a s$ can be seen particularly for larger magnetic fields. It should also be remarked that to get more accurate values of

Table 1. The values of several parameters are tabulated for the case $m=0$ at different suction/injection values.

\begin{tabular}{|c|c|c|c|c|c|c|c|}
\hline$s$ & $\bar{u}_{0}$ & $\bar{v}_{0}$ & $h_{\infty}$ & $r a$ & ras & $h_{\infty a}$ & $\epsilon$ \\
\hline-5 & 0.19756 & $-0 \cdot 01547$ & -0.61925 & 0.07830 & - & - & $85 \cdot 52252$ \\
\hline-3 & $0 \cdot 30914$ & $-0 \cdot 06028$ & -0.67389 & $0 \cdot 19501$ & - & - & $78 \cdot 96480$ \\
\hline-1 & 0.48948 & $-0 \cdot 30217$ & -0.76071 & $0 \cdot 61733$ & - & - & $58 \cdot 31155$ \\
\hline 0 & 0.51023 & -0.61592 & -0.88447 & $1 \cdot 20713$ & - & - & 39.63850 \\
\hline 1 & 0.38956 & $-1 \cdot 17522$ & $-1 \cdot 26055$ & $3 \cdot 01674$ & - & - & $18 \cdot 33950$ \\
\hline 3 & $0 \cdot 16557$ & $-3 \cdot 01214$ & $-3 \cdot 01820$ & $18 \cdot 19169$ & - & - & $3 \cdot 14638$ \\
\hline 5 & 0.09991 & -5.00266 & -5.00399 & $50 \cdot 06986$ & - & - & $1 \cdot 14416$ \\
\hline
\end{tabular}


Table 2. The values of several parameters are tabulated for the case $m=1$ at different suction/injection values.

\begin{tabular}{rccccrrr}
\hline$s$ & $\bar{u}_{0}$ & \multicolumn{1}{c}{$\bar{v}_{0}$} & \multicolumn{1}{c}{$h_{\infty}$} & \multicolumn{1}{c}{$r a$} & \multicolumn{1}{c}{ ras } & \multicolumn{1}{c}{$h_{\infty a}$} & \multicolumn{1}{c}{$\epsilon$} \\
\hline-5 & 0.17788 & -0.20387 & 2.54273 & 1.14615 & -2.13157 & 1.88888 & 41.10416 \\
-3 & 0.24839 & -0.33393 & 1.53137 & 1.34435 & -0.90000 & 1.00000 & 36.64374 \\
-1 & 0.32166 & -0.69066 & 0.43165 & 2.14716 & 1.22727 & 0.11111 & 24.97279 \\
0 & 0.30925 & -1.06905 & -0.25331 & 3.45683 & 3.00000 & -0.33333 & 16.13420 \\
1 & 0.25104 & -1.65707 & -1.08981 & 6.60073 & 5.78571 & -0.77777 & 8.61469 \\
3 & 0.14422 & -3.31056 & -3.01314 & 22.95473 & 22.50000 & -1.66666 & 2.49445 \\
5 & 0.09447 & -5.19480 & -5.00349 & 54.98693 & -94.50000 & -2.55555 & 1.04187 \\
\hline
\end{tabular}

asymptotic counterparts particularly for $|\bar{s}|$ increasing, higher-order terms should be included in the asymptotic expansions (25). The asymptotic mean-flow as calculated above in (25) also implies for large magnetic field that the wave-angle $\epsilon$, especially for large injection cases shrinks considerably as compared to the non-magnetic case. Tables 1-3 show the consistency of our numerical and asymptotic findings.

\subsection{The asymptotic eigenrelation}

Having discussed the properties of the mean-flow, we next concentrate on deriving an analytical estimate for the behaviour of the neutral modes in the large-Reynolds-number limit for any magnetic field and suction parameters $m$ and $\bar{s}$. Applying the zero-slip wall condition to the continuity equation (15) leads to the constraint $W_{0}^{\prime}(0)=0$. This will then generate, considering the solution in (22), the following eigenrelation for the evolution of stationary or non-stationary disturbances

$$
i A \delta^{1 / 2} U_{10}=i A \delta^{3 / 4} \omega_{0} I_{1}+\gamma_{0}^{2} C I_{2}+i \beta_{0} A U^{0^{2}} \bar{u}_{0} I_{3} .
$$

It can be anticipated that the above relation in the absence of both the suction parameter $\bar{s}$ and the magnetic-field parameter $m$ reduces to the non-stationary incompressible lower-branch modes of Turkyilmazoglu (2007), and if further $\omega_{0}$ is set to zero, it reduces to the stationary incompressible lower-branch modes of Hall (1986). Hence, (27) is to calculate modes of neutrally stable state in the magnetohydrodynamic flow over a rotating-disk, accounting for the effects of wall-suction or injection, too. Moreover, a straightforward evaluation from the

Table 3. The values of several parameters are tabulated for the case $m=5$ at different suction/injection values.

\begin{tabular}{rccrrrrr}
\hline \multicolumn{1}{c}{$s$} & \multicolumn{1}{c}{$\bar{u}_{0}$} & \multicolumn{1}{c}{$\bar{v}_{0}$} & \multicolumn{1}{c}{$h_{\infty}$} & \multicolumn{1}{c}{ ra } & \multicolumn{1}{c}{ ras } & \multicolumn{1}{c}{$h_{\infty a}$} & \multicolumn{1}{c}{$\epsilon$} \\
\hline-5 & 0.13193 & -0.85875 & 4.82497 & 6.50907 & -1.18278 & 4.85907 & 8.73415 \\
-3 & $0 \cdot 15143$ & -1.19977 & 2.89628 & 7.92288 & 3.80366 & 2.90351 & 7.19364 \\
-1 & $0 \cdot 15618$ & -1.79957 & 0.95207 & 11.52188 & 10.59314 & 0.94796 & 6.14006 \\
0 & $0 \cdot 14851$ & -2.24345 & -0.02940 & 15.10584 & 15.00000 & -0.02981 & 3.78742 \\
1 & $0 \cdot 13525$ & -2.79707 & -1.01725 & $20 \cdot 67948$ & 20.37942 & -1.00759 & 2.76850 \\
3 & $0 \cdot 10430$ & -4.19539 & -3.00592 & $40 \cdot 22128$ & 35.70853 & -2.96314 & 1.42422 \\
5 & 0.07957 & -5.85538 & -5.00232 & 73.58189 & 63.14999 & -4.91870 & 0.77861 \\
\hline
\end{tabular}


mean-flow equations gives $\bar{u}_{1}=-\frac{1}{2}\left(1+\bar{u}_{0} \bar{s}\right)$ and $\bar{v}_{1}=-\frac{1}{2} \bar{u}_{0} \bar{s}$. Hereafter, for further use, we introduce the notation $\Phi_{0}=\left[\frac{\alpha_{1}}{\beta_{0}}-\frac{\beta_{1} \alpha_{0}}{\beta_{0}^{2}}\right]$. Eventually, decomposing (27) into its real and imaginary parts as well as defining a cut along the negative imaginary semi-axis in the complex plane will result in an equation linking the effective wavenumber $\gamma_{0}$ to frequency $\omega_{0}$ for the development of incompressible neutral waves

$$
a \Omega+b \gamma^{1 / 4}+c \gamma^{9 / 4}=0
$$

where, $\gamma$ and $\Omega$ are the scaled wavenumber and frequencies defined by $\gamma=r^{1 / 2} \gamma_{0}$, $\Omega=r^{-1 / 8} \omega_{0}$. The parameters appearing in equation (28) are also given as $a=$ $\left(\cos \frac{\pi}{8}-\sin \frac{\pi}{8}\right)\left(\frac{r a}{2 U^{0}}\right)^{3 / 4} I_{1}, b=-\bar{u}_{0} U^{0} I_{3}$ and $c=U^{0^{-2}} I_{2}$ with $U^{0}=\left(1+r a^{2}\right)^{1 / 2}$.

Having found the leading-order wavenumber expression, the correction term to the inclination angle, $\Phi_{0}$, can be obtained by utilizing (27), as

$$
\Phi_{0}=\left(\gamma^{3 / 2}+a_{1} \gamma^{-3 / 4} \Omega\right) b_{1} I_{2} r^{-5 / 4}=\phi r^{-5 / 4},
$$

where, the parameters $a_{1}$ and $b_{1}$ are given in Turkyilmazoglu (2007). Moreover, the total effective wavenumber can be written as

$$
k=\sqrt{\alpha^{2}+\frac{\beta^{2}}{r^{2}}}=\gamma_{0}\left[1+\left(\frac{\gamma_{1}}{\gamma_{0}}\right)^{2} \varepsilon^{2}+\cdots\right],
$$

where, $\gamma_{1}$ is to be determined by continuing the higher-order terms, or by simply fixing the wavenumber $\beta_{1}$ and using $\Phi_{0}$ as found in (29). It is straightforward to infer from the relations (29-30) that the influences of non-zero magnetic field come in through the leading-order effective wavenumber term $\gamma$, which is in turn under the strong influence of the suction parameter $\bar{s}$. In a similar fashion, the inclination angle between the radius and crossflow vortices, defined by $\Phi=\arctan \left(\frac{\beta}{\alpha r}\right)$, satisfies

$\cot \Phi=\tan \left(\frac{\pi}{2}-\Phi\right)=\frac{\alpha_{0} r}{\beta_{0}}+\left[\frac{\alpha_{1}}{\beta_{0}}-\frac{\alpha_{0} \beta_{1}}{\beta_{0}^{2}}\right] r \varepsilon^{2}+\cdots=-\frac{\bar{v}_{0}}{\bar{u}_{0}}+\phi r^{-1 / 4} \varepsilon^{2}+\cdots$.

Next investigated are the effects of suction or blowing on the stability features of nonstationary incompressible vortices developing under the influence of a normal uniform magnetic field, by numerically solving the eigenrelation (28) for a range of values $m$ and $\bar{s}$. It can be immediately seen that the zeros of (28) occur at a critical frequency parameter, say $\Omega_{c}$, which might be evaluated as $\Omega_{c}=\frac{8 c}{a}\left(\frac{-b}{9 c}\right)^{9 / 8}$, and hence the corresponding wavenumber from (28) is $\gamma_{c}=\frac{\sqrt{\frac{-b}{c}}}{3}$. It is thus easy to judge that whenever $\Omega>\Omega_{c}$ no modes can exist for any fixed values of $m$ and $\bar{s}$, otherwise multiple modes appear. As a result of this, $\Omega_{c}$ may be considered as a threshold value for the frequency (which happens to be always positive) for the non-stationary incompressible modes to appear for a given suction parameter $\bar{s}$. From the asymptotic relations in (25), it is readily obtained for large magnetic-field parameter that $\left(\Omega_{c}, \gamma_{c}\right) \sim\left(\frac{8 I_{2}}{9 \hat{a}}\left(I_{3} / I_{2}\right)^{9 / 8} m^{13 / 16},\left(I_{3} / I_{2}\right)^{1 / 2} m^{5 / 4}\right)$. Hence the occurrence of multiple modes is possible for large values of $m$, regardless of the existence of suction or blowing. Similarly, a large-suction analysis for fixed values of $m$ produces $\left(\Omega_{c}, \gamma_{c}\right) \sim\left(\bar{s}^{13 / 8}, \bar{s}^{5 / 2}\right)$, whereas for large-blowing limit $\left(\Omega_{c}, \gamma_{c}\right) \sim 0$. This points to the disappearance of the multiple modes for large-blowing limit at any fixed (but finite) magnetic-field parameter $m$. It should also be remarked here that, negative or complex roots of (28) have also been encountered for 

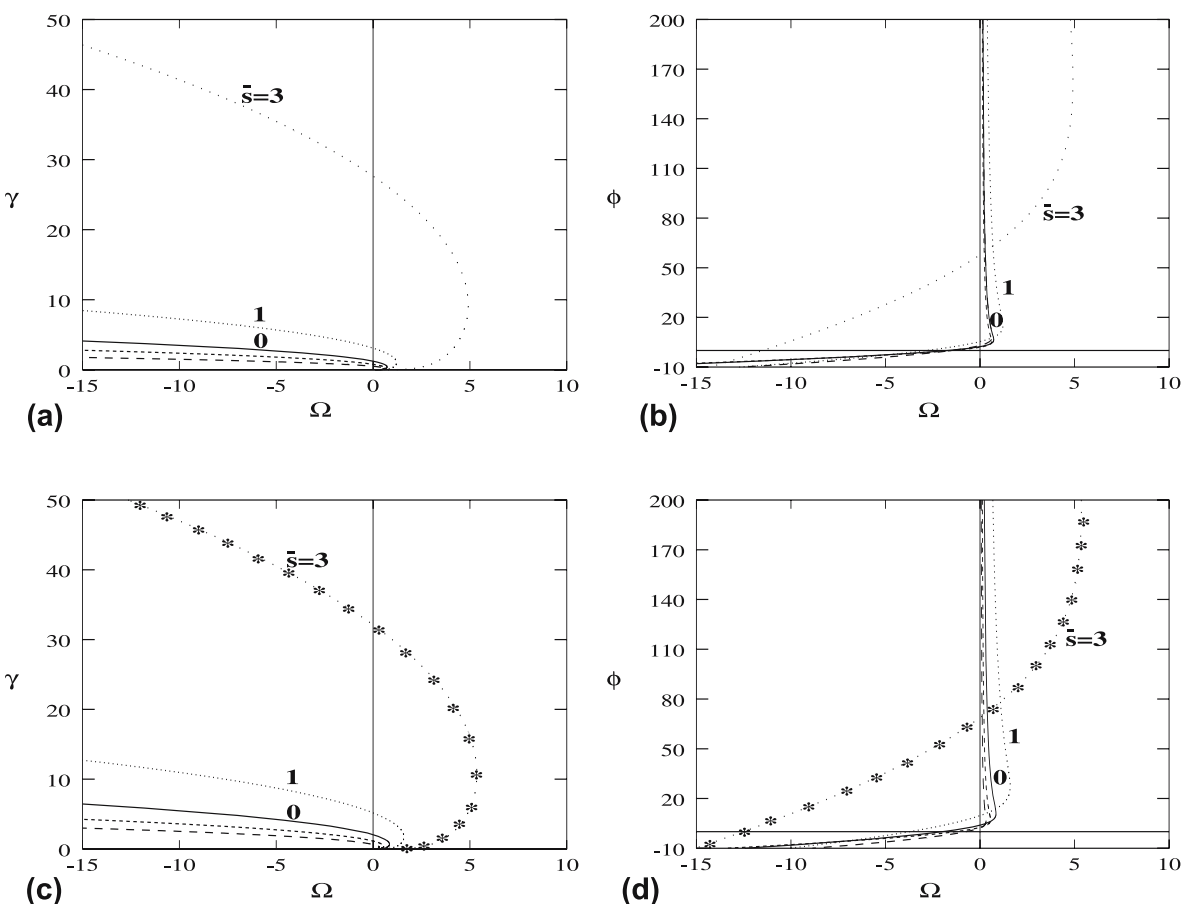

Figure 4. The evolution of non-stationary neutral leading-order wavenumber (a-c) and wave-angle (b-d) against the frequency are demonstrated for different suction parameters, respectively, $\bar{s}=3(\ldots), \bar{s}=1(\ldots), \bar{s}=0(-), \bar{s}=-1(--)$ and $\bar{s}=-3(--)$. First figures are for the magnetic-strength parameter $m=0$ and the second are for $m=0.5$ respectively. The small magnetic field perturbation approach (see the appendix) results in the solutions denoted by starred curves in parts (c-d).

the scaled wavenumber $\gamma$. Taking into consideration the definition of $\gamma$, negative values are physically unrealistic. On the other hand, the discussion of complex $\gamma$ is beyond the scope of this work, since we concentrate here on the neutral modes only. However, the complex roots may give amplifying/damped non-stationary waves whose validity need to be verified by a further linear stability analysis of non-neutral modes.

In figures 4-6 the scaled leading-order wavenumber $\gamma$ and the scaled wave-angle correction $\phi$ are demonstrated against the frequency to point out the effects of magnetic field on the evolution of stationary/non-stationary incompressible lower-branch neutral modes accounting for both the suction as well as blowing cases. It is immediately seen that increasing the magnetic-field parameter much influences progression of the lower-branch modes, particular emphasis should be placed on the larger values of $m$. It can thus be deduced from figures 4-5 that as $m$ increases from the non-magnetic case, the wavenumber and wave-angle correction terms are also increasing if the suction is applied on the wall, as compared to the nonmagnetic-field case. However, if the blowing is active on the surface, even though a similar trend to the suction case appears for $\gamma$, the wave-angle correction parameter $\phi$ substantially decreases for increasing $m$, particularly for negative frequencies. Overall, the wave-angle which vortices make with the radius vector is shown to lie in a wide range $\left[0^{\circ}, 90^{\circ}\right]$. When the fluid is non-conducting, then the trends of the eigenvalues are seen to be in complete consistence with the zero-suction stationary/nonstationary incompressible disturbances as found 


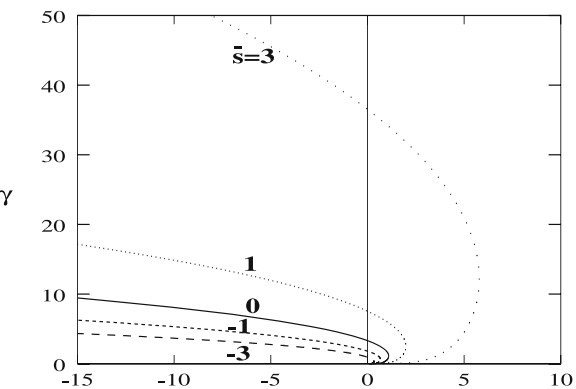

(a)

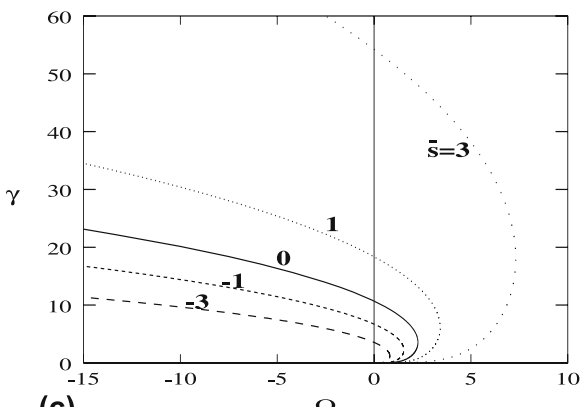

(c)

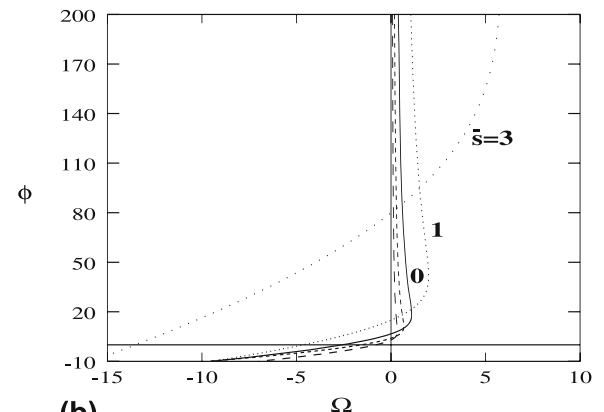

(b)

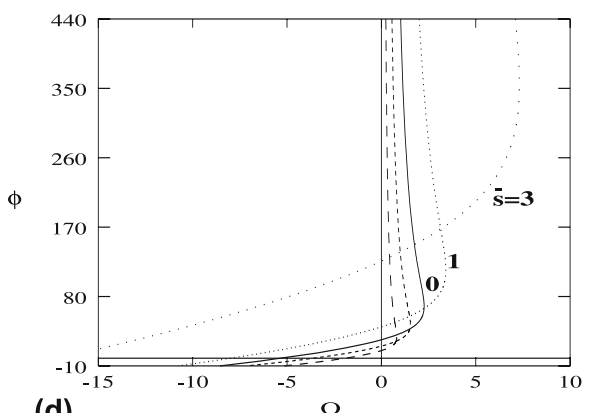

(d)
$\Omega$

Figure 5. The evolution of non-stationary neutral leading-order wavenumber (a-c) and wave-angle (b-d) against the frequency are demonstrated for different suction parameters, respectively, $\bar{s}=3(\ldots), \bar{s}=1(\ldots), \bar{s}=0(-), \bar{s}=-1(--)$ and $\bar{s}=-3(--)$. First figures are for the magnetic-strength parameter $m=1$ and the second are for $m=3$ respectively.

in Turkyilmazoglu (2007), and varying suction results of Turkyilmazoglu (2005). It should also be noticed that the presented results are in excellent agreement with the asymptotic results for large-and for small-perturbation results of magnetic flow field cases, which are respectively demonstrated in figures 6 and 4(c-d) at some chosen suction and magnetic-field parameters.

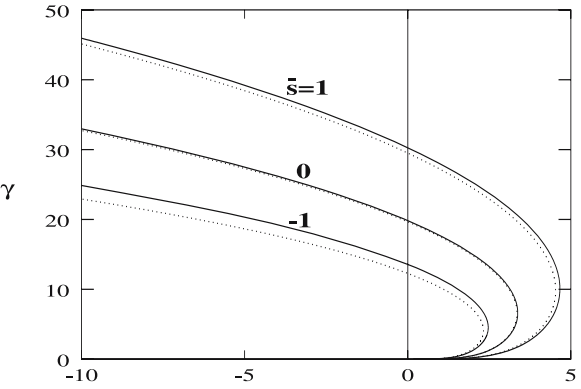

(a)

$\Omega$

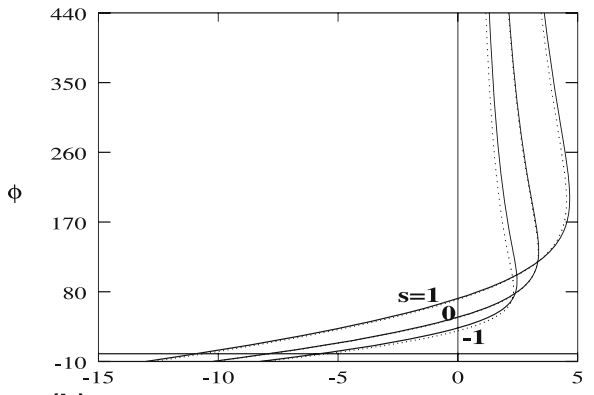

(b)

Figure 6. The evolution of non-stationary neutral leading-order wavenumber (a) and wave-angle (b) against the frequency at the chosen large magnetic-strength parameter $m=5$ are demonstrated for two selected suction parameters. Dots for each curve correspond to the asymptotic large-magnetic-field limit referred to in the text. 
Fully in line with the findings of Dhanak (1992) for the incompressible non-conducting rotating-disk boundary-layer flow using linear stability theory, the powerful stabilization impact of the suction is apparent from figures 4-6, unlike the destabilization effect of blowing. Hence, as expected from the result of Jasmine \& Gajjar (2005), one of the main conclusion from the current research is that the magnetic-field effects are always in favor of stabilizing the rotating-disk boundary-layer flow by means of enlarging the wavenumber regime. What is intriguing is that the stabilizing impact is more pronounced in the fluid suction case, while a weak persistence seems to exist for fluid injection case. Another important conclusion to be made from figures 4-6 is that as compared to the non-magnetic incompressible flow case, the perturbations introduced into the magnetic incompressible boundary-layer will develop on a smaller wavelength, even much smaller in the case of suction as the magnetic field gets stronger, despite the fact that the injection case persists in this situation. Moreover, disturbances have, regardless of the suction or blowing, longer wavelength for non-stationary positive-frequency waves than for the non-stationary negative-frequency waves, in line with the outcomes of Turkyilmazoglu (2007) for zero-suction case and also of Turkyilmazoglu (2005) for non-zero-suction case. This in turn indicates that an unstable mode of instability is more likely to occur for an incompressible conducting flow having positive frequencies. This outcome is in compliance with the experimental results of Jarre \& Chauve (1996a,b) in the case of non-magnetic-field case, but similar experiments including the stationary/travelling disturbances in the conducting fluid case are needed to justify the present outcome.

One can further observe from figures 4-6 that, as mentioned before, multiple modes exist for the non-stationary lower-branch neutral modes in the interval $\left[0, \Omega_{c}\right]$, which is not the case for the stationary waves of Hall (1986) and Seddougui \& Bassom (1996). The evidence for the existence of such a mode in the incompressible non-stationary waves was first provided in the numerical calculations of Balakumar \& Malik (1990). The impact of the presence of magnetic field is to increase the interval of double modes for positive frequencies as $m$ increases, an effect that is in line with the former asymptotic findings. The increasing effect is more effective in the wall-suction case, but for a fixed finite magnetic-field parameter it is also true that large injection is capable of removing the existence of multiple modes, since from the large wall-injection asymptotic limit $\Omega_{c} \rightarrow 0$.

And finally, using the large asymptotic limiting behaviours of the mean-flow, estimates can be derived in the case of injection for fixed finite $m$ and small $\gamma, \Omega \sim(-\bar{s})^{1 / 2} \gamma^{1 / 4}$ and for $\gamma$ large and fixed $\Omega \sim-\infty$, while in the case of suction for fixed finite $m$ the behaviours will result in $\Omega \sim \bar{s} \gamma^{1 / 4}$ and $\Omega \sim-(\bar{s})^{-4} \gamma^{9 / 4}$, respectively. On the other hand, in the case of large magnetic field for fixed finite $\bar{s}, \Omega \sim m^{1 / 2} \gamma^{1 / 4}$. Particularly for the stationary modes for large magnetic field the leading-order wavenumbers and angle can be found as $\gamma=3 \sqrt{I_{3} / I_{2}} m^{5 / 4}$, $\beta_{0}=\sqrt{I_{3} / I_{2}} m^{1 / 4} r^{1 / 2}, \alpha_{0}=3 \sqrt{I_{3} / I_{2}} m^{5 / 4} r^{-1 / 2}$ and $\phi=\cot ^{-1}(3 m)$. These asymptotic limits are fully consistent with the results displayed in figures $4-6$.

\subsection{Comparison with the parallel-flow approximation}

Numerical results of parallel-flow approximation within the framework of classical linear stability theory were given in Balakumar \& Malik (1990) for zero-suction and in Dhanak (1992) for suction or injection both for the incompressible Karman flow of a non-conducting fluid case. In the present case we want to compare the asymptotic findings of magnetic field with the ones obtained from the linear stability theory, particulary with those of Jasmine \& Gajjar (2005) for zero-suction case. However, to make a proper comparison between the present asymptotic results as found in section 4.2 with the parallel-flow results of Jasmine \& Gajjar (2005) and Dhanak (1992), new parameters $R_{\Delta}, k_{\Delta}$ and $\omega_{\Delta}$ need to be introduced 

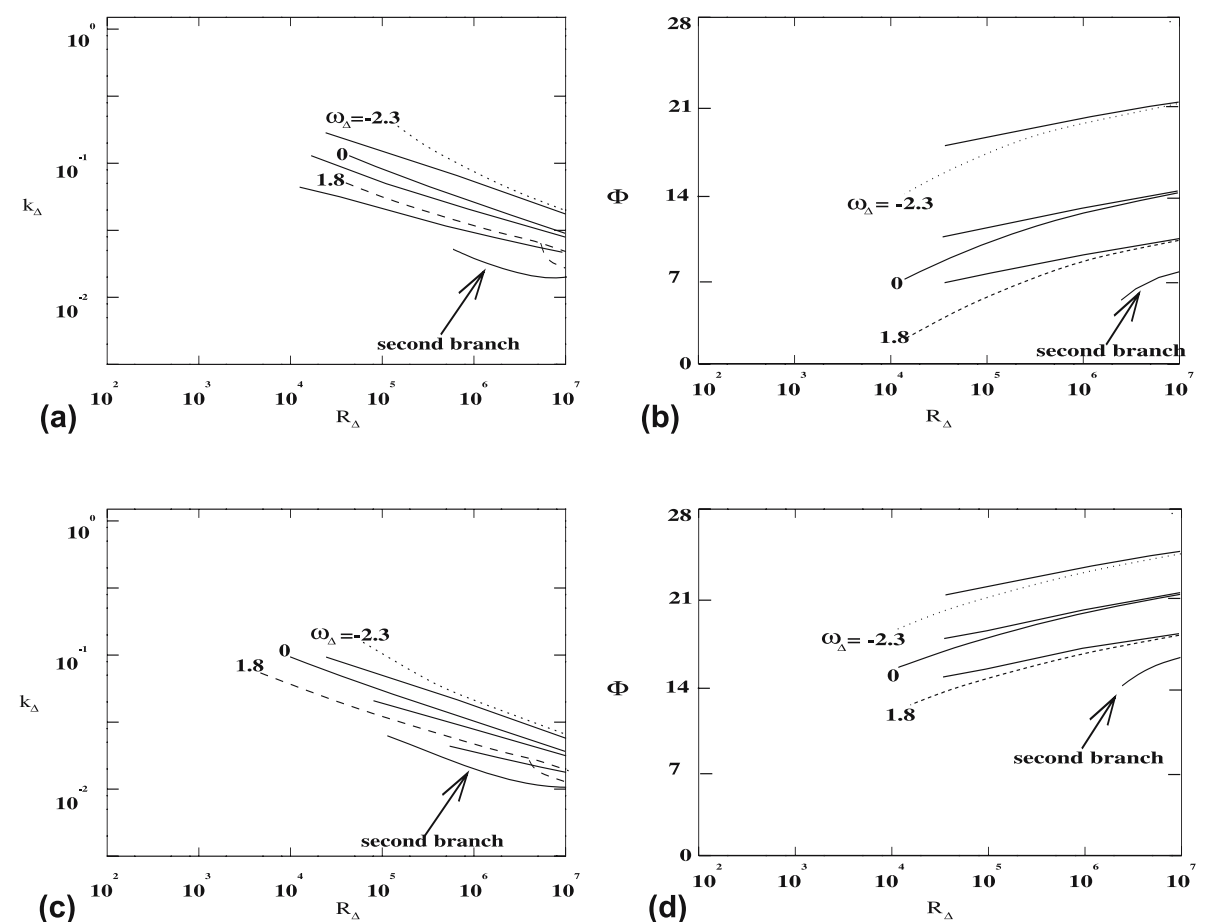

Figure 7. The effects of magnetic field with $m=0.5$ are illustrated of the variation of effective wavenumber $(\mathbf{a}, \mathbf{c})$ and wave-angle $(\mathbf{b}, \mathbf{d})$ against the Reynolds number for a variety of frequencies $\omega_{\Delta}=-2.3(\ldots), 0(-)$ and $1.8(--)$. First pair is for the suction $s=1$, and the second is for the injection $s=-1$. The asymptotic structure of the non-stationary lower-branch modes (non-parallel) is also shown by the unbroken curves corresponding to each frequency.

first, which are mainly the Reynolds number, wave number and frequency respectively. The relations of these to $\gamma, \phi$ and $\Omega$ are given in Turkyilmazoglu (2007). In terms of these quantities, the incompressible linear stability equations of conducting fluid flow were solved employing a Chebyshev Collocation technique whose details may be found in Turkyilmazoglu (1998). In figures 7-8 a comparison has been made between the computed numerical results of parallel-flow approximation and the non-parallel asymptotic predictions (28-30) to investigate the evolution of stationary/non-stationary lower-branch modes with both the magnetic field and suction/injection in effect.

At two magnetic fields, the influences of suction and injection are demonstrated in figures 7-8. The presence of magnetic field is seen towards stabilization for both suction and injection cases. Moreover, it is seen that the destabilizing effect of the non-parallelism (unlike the stabilizing effect of it on the open shear flows such as mixing layers and jets) is found to be greater when injection is applied, since a larger extend of the wavenumber domain undergoes instability, as opposed to the case of suction in line with the experiment of Gregory \& Walker (1960) for the non-conducting fluid flow. Again, in parallel to the outcomes of section 4.2, together with the magnetic field, suction is more stabilizing as compared to the case of injection. The second mode discovered from the asymptotic analysis is also shown in figures 7-8 for the positive frequency $\omega_{\Delta}=1 \cdot 8$. Such a mode is of a practical importance since for large-amplitude disturbances these modes may bifurcate sub-critically if the non-linearity is taken into account, see for example, Mackerrel (1987). 

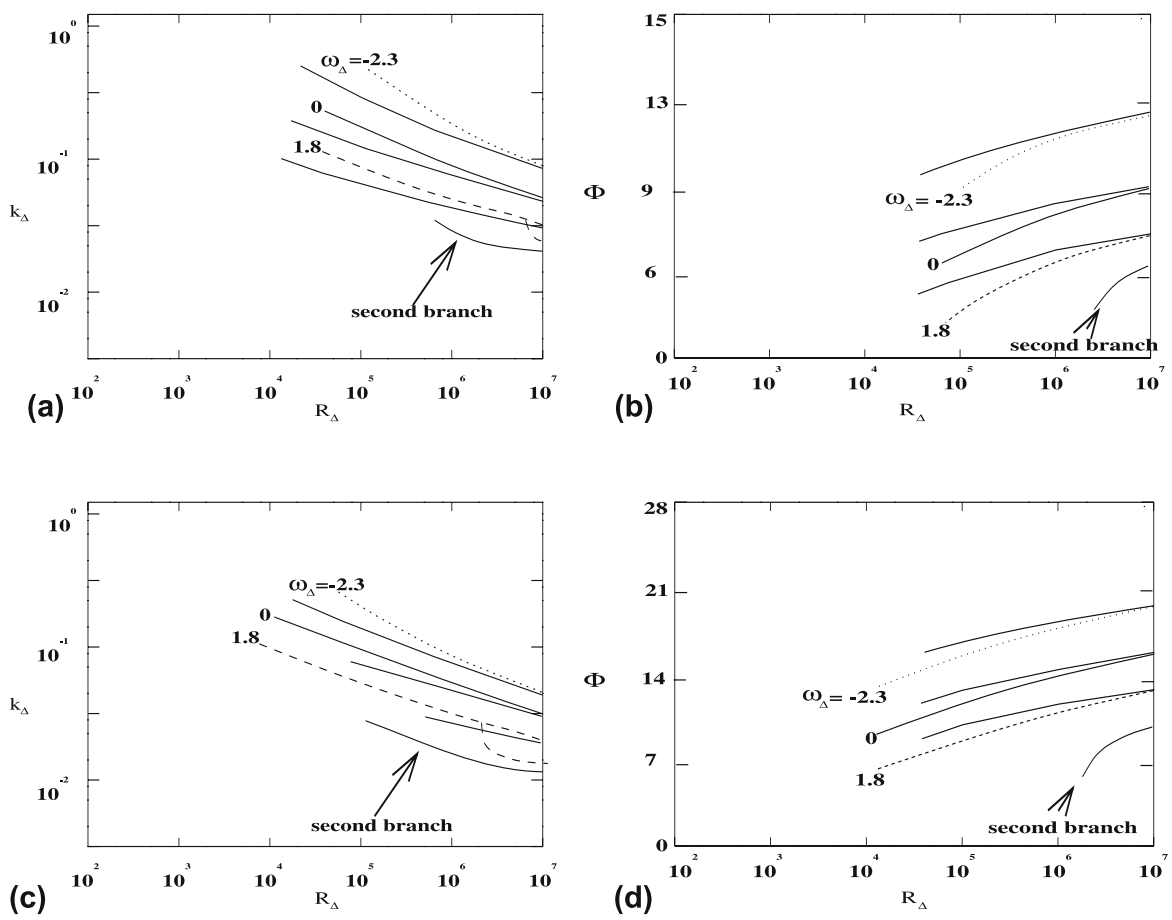

Figure 8. The effects of magnetic field with $m=1$ are illustrated of the variation of effective wavenumber $(\mathbf{a}, \mathbf{c})$ and wave-angle $(\mathbf{b}, \mathbf{d})$ against the Reynolds number for a variety of frequencies $\omega_{\Delta}=-2.3(\ldots), 0(-)$ and $1.8(--)$. First pair is for the suction $s=1$, and the second is for the injection $s=-1$. The asymptotic structure of the non-stationary lower-branch modes (non-parallel) is also shown by the unbroken curves corresponding to each frequency.

It should also be noted that asymptotes shown by the unbroken curves in figures 7-8 represent only a first-order correction to the inviscid mode. To get refined approximations for practically interesting Reynolds numbers more terms in the expansions (30-31) should be accounted for so that a better comparison by the parallel-flow approximation of Jasmine \& Gajjar (2005) can be done.

\section{Conclusions}

A self-consistent and rigorous asymptotic expansion technique based on the well-known triple-deck theory has been made use in this study to search for the details of the behaviour of the short wavelength small-frequency lower-branch neutral modes, with emphasis placed upon non-stationary disturbances imposed on the incompressible three-dimensional boundarylayer flow over a rotating-disk in the presence of a uniform magnetic field applied perpendicular to the disk surface. The consideration of a conducting fluid together with the imposition of suction or blowing on the boundary-layer has a substantial effect on the mean-flow quantities, which in turn has a great influence on the stability properties of this boundary-layer. The investigation has been extended to visualize the differences present in the solution of the linearized system of the incompressible magnetic flow equations obtained by employing the traditional parallel-flow assumption and the asymptotically determined ones, for both the suction and injection applied. 
It has been shown that a second mode appears for some small-frequency waves whose domain of definition increases indefinitely in the presence of a large-enough magnetic field. The domain further increases with suction but it decreases with injection. Moreover, a critical value depending strongly on the magnetic-field and suction parameters has been calculated asymptotically, which puts restrictions on the existence of positive-frequency modes. Further, the non-parallelism coming into effect owing to the second-order terms in the asymptotic expansions has been found to be destabilizing making the flow more convectively unstable, though unimportant within the strong stabilizing effect of especially large magnetic field. Sucking the boundary-layer under the influence of a wall-normal magnetic field has an extra stabilizing effect, though fluid injection seems to resist the stabilization. Hence the main conclusion from the present work is that the presence of a normal magnetic field is stabilizing as compared to the non-magnetic case, which is apparently due to the reduction effect of the magnetic flow on the mean-flow quantities. To make a comparison between the stationary and non-stationary waves at a fixed magnetic field as well as suction parameter, the positivefrequency modes have been shown to be more destabilized as compared to the waves having negative frequencies. Thus, the zero-frequency neutral waves have been shown to play a role of separating non-zero-frequency waves.

The present asymptotic results obtained within the large-Reynolds-number approximation have been also compared with those of obtained from the usual parallel-flow approximation to the full linearized stability equations, which were formulated here for the conducting fluid over the rotating-disk boundary-layer flow. The influences of magnetic field as well as the suction/injection through the surface of the disk have been demonstrated to be well captured from the both approaches.

Further effects of temperature-dependent viscosity together with the magnetic field are also of physical interest. How the non-linearity may influence the non-stationary modes computed here is also of great significance, as for the stationary modes of Mackerrel (1987) and Seddougui \& Bassom (1996). Finally, other forms of the magnetic field are also worthy of extra consideration.

\section{A. Appendix}

In this appendix, a perturbation solution to mean-flow for small magnetic-field parameter $m$ with fixed and finite $\bar{s}$ will be presented. For small $m$, the solution can be assumed of the perturbation of the parameter $m$, thus

$$
F=F_{0}+m F_{1}+\cdots, G=G_{0}+m G_{1}+\cdots, H=H_{0}+m H_{1}+\cdots .
$$

Substituting above expansions into the mean-flow equations (7-9) leads to following nonlinear equations, respectively $O(1), O(m)$ and $O\left(m^{2}\right)$

$$
\begin{aligned}
& F_{0}^{2}-\left(G_{0}+1\right)^{2}+F_{0}^{\prime} H_{0}-F_{0}^{\prime \prime}=0, \\
& 2 F_{0}\left(G_{0}+1\right)+G_{0}^{\prime} H_{0}-G_{0}^{\prime \prime}=0, \\
& 2 F_{0}+H_{0}^{\prime}=0, \\
& 2 F_{0} F_{1}-2\left(G_{0}+1\right) G_{1}+F_{0}^{\prime} H_{1}+H_{0} F_{1}^{\prime}-F_{1}^{\prime \prime}=-F_{0}, \\
& 2 F_{0} G_{1}+2\left(G_{0}+1\right) F_{1}+G_{0}^{\prime} H_{1}+H_{0} G_{1}^{\prime}-G_{1}^{\prime \prime}=-\left(G_{0}+1\right), \\
& 2 F_{1}+H_{1}^{\prime}=0,
\end{aligned}
$$




$$
\begin{aligned}
& 2 F_{0} F_{2}-2\left(G_{0}+1\right) G_{2}+F_{0}^{\prime} H_{2}+H_{0} F_{2}^{\prime}-F_{2}^{\prime \prime}=-\left(F_{1}^{2}+F_{1}-G_{1}^{2}+F_{1}^{\prime} H_{1}\right), \\
& 2 F_{0} G_{2}+2\left(G_{0}+1\right) F_{2}+G_{0}^{\prime} H_{2}+H_{0} G_{2}^{\prime}-G_{2}^{\prime \prime}=-\left(G_{1}+2 F_{1} G_{1}+G_{1}^{\prime} H_{1}\right), \\
& 2 F_{2}+H_{2}^{\prime}=0 .
\end{aligned}
$$

Equations are considered with suitable boundary conditions obtained from equation (9). It can be seen that equations (32) are coupled and non-linear by nature for all the values of suction parameter $\bar{s}$ and the solution of which is not possible analytically. A similar situation prevails for the subsequent sets (33-34). Therefore, numerical solutions to (32-33) are obtained.

\section{References}

Abramowitz M, Stegun I A 1955 Handbook of mathematical functions (New York: Dover Publications) Attia H A 2003 Time varying rotating disk flow and heat transfer of a conducting fluid with suction or injection. Int. Comm. Heat Mass Transfer 30: 1041-1049

Balakumar P, Malik M R 1990 Travelling disturbances in rotating-disk flow. Theoret. Comput. Fluid Dyn. 2: 125-137

Bassom A P, Gajjar J S B 1988 Non-stationary crossflow vortices in a three dimensional boundary layer. Proc. Roy. Soc. London Ser. A 417: 179-212

Bassom A P, Seddougui S O 1992 The effects of suction on the non-linear stability of the threedimensional boundary layer above a rotating disc. Proc. Roy. Soc. London Ser. A 436: 405

Benton E T 1966 On the flow due to a rotating disk. J. Fluid Mech. 24: 781-800

Bhatnagar P L, Banerjee M B 1978 On the hydrodynamic and hydromagnetic stability of superposed fluids. J. Math. Phys. Sci. 12: 267-286

Chandrasekkar S 1961 Hydrodynamic and hydromagnetic stability. Oxford University Press

Chang M H 2003 Hydrodynamic stability of taylor-dean flow between rotating porous cylinders with radial flow. Phys. Fluids. 15: 1178-1188

Cole J W 1995 Hydrodynamic stability of compressible flows. Ph.D. thesis, University of Exeter

David R, Georges G, Daniel B 2005 Instabilities between rotating and stationary parallel disks with suction. Phys. Fluids. 17: 181-185

Davies C, Carpenter P W 2003 Global behaviour corresponding to the absolute instability of the rotating-disc boundary layer. J. Fluid Mech. 486: 287-329

Dhanak M R 1992 Effects of uniform suction on the stability of flow on a rotating disc. Proc. Roy. Soc. London Ser. A 439: 431

Gregory N, Stuart J T, Walker W S 1955 On the stability of three dimensional boundary layers with applications to the flow due to a rotating-disk. Philos. Trans. R. Soc. London Ser. A 248: 155-199

Gregory N, Walker W S 1960 Experiments on the effects of suction the flow due to a rotating disk. J. Fluid Mech. 9: 225

Hall P 1986 An asymptotic investigation of the stationary modes of instability of the boundary layer on a rotating-disk. Proc. Roy. Soc. London Ser. A 406: 93-106

Hossain M A, Hossain A, Wilson M 2001 Unsteady flow of viscous incompressible fluid with temperature-dependent viscosity due to a rotating disc in the presence of transverse magnetic field and heat transfer. Int. J. Therm. Sci. 40: 11-20

Jarre S L G, Chauve M P 1996a Experimental study of rotating-disk instability. I. Natural flow. Phys. Fluids. 8: 496-508

Jarre S L G, Chauve M P 1996b Experimental study of rotating-disk instability. II. Forced flow. Phys. Fluids. 8: 2985-2994

Jasmine H A, Gajjar J S B 2005 Convective and absolute instability in the incompressible boundary layer on a rotating disk in the presence of a uniform magnetic field. Journal of Engineering Mathematics 52: 337-353 
Kafoussias N G, Nanousis N D 1999 Magnetohydrodynamic laminar boundary-layer flow over a wedge with suction or injection. Can. J. Phys. 75: 733-745

Kármán T V 1921 Uber laminare und turbulente Reibung. Zeitschift f́' ur angewantee Mathematik und Mechanik, 1: 233-252

Lingwood R J 1995 Absolute instability of the boundary layer on a rotating-disk. J. Fluid Mech. 299: $17-33$

Lingwood R J 1996 An experimental study of absolute instability of the rotating-disk boundary layer flow. J. Fluid Mech. 314: 373-405

Lingwood R J 1997 On the effects of suction and injection on the absolute instability of the rotatingdisk boundary layer. Phys. Fluids. 9: 1317-1328

Mackerrel S O 1987 A nonlinear asymptotic investigation of the stationary modes of instability of the 3 dimensional boundary layer on a rotating-disk. Proc. Roy. Soc. London Ser. A 413: 497-513

Malik M R 1986 The neutral curve for stationary disturbances in rotating-disk flow. J. Fluid Mech. 164: 275-287

Malik M R, Poll D I A 1985 Effect of curvature on three dimensional boundary layer stability. AIAA Journal 23: 1362-1369

Malik M R, Wilkinson S P, Orszag S A 1981 Instability and transition in rotating-disk flow. AIAA Journal 19: 1131-1138

Othman H 2005 Experimental study of absolute instability of a rotating-disk boundary layer. Ph.D. thesis, Univ. of Notre Dame, Dept. of Aerospace \& Mech. Eng.

Othman H, Corke T C 2005 Experimental investigation of absolute instability of a rotating-disk boundary layer. submitted to. J. Fluid Mech.

Pao H P 1968 Magnetohydrodynamic flows over a rotating disk. AIAA Journal 6: 1285-1291

Pier B 2003 Finite-amplitude crossflow vortices, secondary instability and transition in the rotatingdisk boundary layer. J. Fluid Mech. 487: 315-343

Seddougui S O 1990 A nonlinear investigation of the stationary mode of instability of the three dimensional compressible boundary layer due to rotating-disk. Q. J. Mech. Appl. Math. 43: 467-497

Seddougui S O, Bassom A P 1996 The effects of suction on the non-linear stability of a threedimensional compressible boundary layer. IMA J. Applied Math. 56: 183

Sparrow E M, Cess R D 1962 Magnetohydrodynamic flow and heat transfer about a rotating disk. J. Appl. Mech. 29: 181-187

Sparrow E M, Gregg J L 1959 Heat transfer from a rotating disk to fluids of any Prandtl number. J. Heat Transfer 81: 249-251

Stewartson K 1969 On the flow near the trailing-edge of a flat plate. Mathematica 16: 106-121

Stewartson K, Williams P G 1969 Self-induced separation. Proc. Roy. Soc. London Ser. A 312: 181-206

Stuart J T 1954 On the effects of uniform suction on the steady flow due to a rotating disk. Q. J. Mech. Appl. Math. 7: 446-457

Turkyilmazoglu M 1998 Linear absolute and convective instabilities of some two- and three dimensional flows. Ph.D. thesis, University of Manchester

Turkyilmazoglu M 2005 Effects of suction on the non-stationary lower branch modes of a compressible boundary layer flow due to a rotating disk. Stud. Appl. Math. 115: 357-385

Turkyilmazoglu M 2007 Non-linear and non-stationary modes of the lower branch of the incompressible boundary layer flow due to a rotating-disk. Quart. Appl. Math. 65: 43-68

Turkyilmazoglu M, Cole J W, Gajjar J S B 2000 Absolute and convective instabilities in the compressible boundary layer on a rotating disk. Theoret. Comput. Fluid Dyn. 14: 21-37

Turkyilmazoglu M, Gajjar J S B 2001a An analytic approach for calculating absolutely unstable inviscid modes of the boundary layer on a rotating-disk. Stud. Appl. Math. 106: 419-435

Turkyilmazoglu M, Gajjar J S B 2001b Upper branch non-stationary modes of the boundary layer due to a rotating-disk. Applied Math. Lett. 14: 685-690

Turkyilmazoglu M, Uygun N 2005 Compressible modes of the rotating disk boundary layer flow leading to absolute instability. Stud. Appl. Math. 115: 1-20 